\title{
INTERVENTION EFFECT OF SENSORY INTEGRATION TRAINING ON THE BEHAVIORS AND QUALITY OF LIFE OF CHILDREN WITH AUTISM
}

\author{
Wenxin $\mathrm{Xu}^{1}$, Jiwei $\mathrm{Yao}^{2}$ \& Wenyao Liu ${ }^{3}$ \\ ${ }^{1}$ Institute of Physical Education and Sport Science, Fujian Normal University, Fuzhou, Fujian, China \\ ${ }^{2}$ Physical Education Institute, Hunan University of Science and Technology, Xiangtan, Hunan, China \\ ${ }^{3}$ Fujian Normal University Hospital, Fuzhou, Fujian, China
}

received: 16.8.2019;

revised: 4.9.2019;

accepted: 11.9.2019

\begin{abstract}
SUMMARY
Background: Autism is a widespread developmental disorder that occurs mostly among children. Children with autism are prone to problematic behaviors due to their deficiencies in language communication and social development. Thus, children with a high degree of autism suffer lower life satisfaction. Moreover, sensory integration dysfunction is closely related to autism. Therefore, the effect of Sensory Integration Training (SIT) on the behaviors and quality of life of children with autism was explored in this study.

Subjects and methods: From September 2017 to December 2018, 108 patients from Fuzhou Fourth Hospital and Xiangtan Fifth Hospital were included in the intervention group (group A) and the control group (group B), with 54 members in each group. The 54 members in group $B$, with an average age of $5.18 \pm 2.94$, received routine treatment. In addition to the same routine treatment, the members in group $B$ also received sensory integration training and physical exercise intervention, which lasted for three months. The Childhood Autism Rating Scale (CARS) and Autism Behavior Checklist (ABC) were used before and after the intervention experiment to evaluate the curative effect.

Results: After the treatment, statistically significant differences were observed in the CARS and ABC scores $(P<0.05)$; the total effective rate was $86.11 \%$ in group $A$ and $64.10 \%$ in group $B$. The difference in the CARS score was statistically significant $(P<0.05)$, whereas the difference in the ABC score was also statistically significant $(P<0.05)$. In general, the difference in CARS is statistically significant. Specifically, group $A$ is better than group $B, t=3.492, d f=73$, and bilateral $P=0.001<0.01$.

Conclusions: SIT intervention had a certain effect on autism and is of great value for the future development of SIT courses or intervention programs for children with autism.
\end{abstract}

Key words: sensory integration training - SIT - autism - problematic behaviors - quality of life

\section{INTRODUCTION}

Autism Spectrum Disorder, also known as autism, is a neurodevelopmental disorder that usually occurs in early childhood development (Dawson et al. 2010). The main features of this spectrum disorder are impairment in children's social and communication functions, repetitive behaviors, and peculiar interests (Morales-Hidalgo et al. 2018). A US prevalence survey in 2014 stated that one in every 69 children aged 8-11 years old is diagnosed with autism (Morales-Hidalgo et al. 2018). This proportion has considerably risen to one in every 60 children (Christensen et al. 2016). Thus, the prevalence of autism in children is high and should be a general concern to all sectors of society especially relevant educators (Chandler et al. 2016). Owing to the influence of autism, the physical, mental health, and quality of life of children with autism are not optimistic (Xu et al. 2018). Bruggink et al. (2016) concluded that no significant difference was observed between children with autism and children without autism. in the number of emotional adjustment strategies they have utilized. However, the nature of these strategies have essential differences. The former tends to use the emotional ad- justment strategy of blaming others wherein their level of depression and anxiety was observed to be significantly higher than children without autism. Mccoy et al. (2016) investigated a total of 42,747 adolescents, among which 915 adolescents were autistic patients. The analysis established that the intensity of autism was positively correlated with the degree of obesity in adolescents. Adolescents with a high degree of autism are less likely to participate in various physical exercises, teambased activities, or club activities. Barnett \& Crippen (2014) stated that the life satisfaction of children with autism is significantly lower than that of children without autism. Thus, a high degree of autism results in low life satisfaction. In sum, researchers should focus on the physical and mental health as well as the quality of life of children with autism.

To improve the mental health and behavioral level of children with autism, numerous researchers have conducted intervention studies from different perspectives. For instance, Luxford et al. (2017) randomized 35 children with autism, including 18 in the intervention group and 17 in the control group. Intervention targeting six modules of cognitive behaviors was conducted in the intervention group. After the intervention, children with 
autism in the intervention group exhibited reduced anxiety symptoms and improved social responsiveness. Researchers also intervened to improve the social skills of children with autism and achieved some positive results (Gates et al. 2017). Although psychosocial behavior-related interventions provide a better idea for intervening in children with autism and are effective interventions to a certain extent, these methods seem to ignore that children with autism have behavioral problems such as physical disharmony. Therefore, an increasing number of researchers have begun to pay attention to the role of Sensory Integration Training (SIT) in the treatment of behavioral problems in children with autism. SIT can effectively improve the behavioral capacity and social interests of children with autism and enhance their social functions (Lang et al. 2012).

In summary, experts in different fields have proposed different interventions for children with autism. These interventions can be generally seen in three directions: psychology, education, and medicine. Exercise intervention belongs to the field of education, because exercise itself has educational function. Through exercise, one can correct bad behavioral habits, enhance will, and overcome difficulties (Kim \& Jeon 2017, Xu et al. 2016). Different exercise interventions have been designed according to the situations of sensory integration dysfunction in children with autism, because the body can constantly receive different external stimuli through exercise. The eyes, ears, skin, and nose are fully utilized during exercise. Rich sensory information flows into the brain and activates nervous systems, thereby promoting the development of various nervous systems and improving the brain's sensory integration ability (Cheldaviet al. 2014). Scholars believed that sensory integration dysfunction is closely related to autism, and the symptoms of sensory integration dysfunction are similar to those of autism (Wu 2011). Although domestic researchers have also investigated the influence of SIT on the ability to balance, ability to exercise ability (Dai $\&$ Ma 2008), stereotypes and sensory disorders (Chen et al. 2017) of children with autism, only a few comprehensive investigations have been conducted on the effect of SIT on the behaviors and quality of life of children with autism. Therefore, this innovative study on the joint effect of SIT and exercise intervention on the behaviors and quality of life of children with autism can provide further significant clinical reference for improving the negative behaviors of children with autism.

\section{SUBJECTS AND METHODS}

\section{Participants}

From September 2017 to December 2018, a total of 108 participants from Fuzhou Fourth Hospital and Xiangtan Fifth Hospital were included according to the diagnostic criteria. The consent of participants and their guardians was obtained prior to the study. Participants in the study were informed about the intervention method and the purpose and significance of the study. Thus, they all participated in the study voluntarily. The diagnostic criteria are from the $3^{\text {rd }}$ edition of Classification and Criteria of Mental Disorders (CCMD-3) (Li et al. 2002). Inclusion criteria include the following:

- those who meet the diagnostic criteria;

- aged 2-14 years old;

- those who could correctly and seriously deal with the study with good compliance;

- those who are willing to participate in the study voluntarily (informed consent is required). Exclusion criteria:

- those who do not meet the diagnostic criteria;

- those under 2 years old or above 14 years old;

- those with severe systemic diseases;

- those who are unwilling to participate in the study.

The participants in this experiment had already understood the purpose and method of the experiment before they proceeded, and all of them participated voluntarily in the study.

\section{Methods}

The random method was adopted. The 108 patients who participated in the experiment were randomly divided into group A (SIT + routine treatment group) and group B (routine treatment group) after signing the experiment agreement. Each group had 54 members. Group B was treated with routine treatment, such as education and psychotherapy; whereas, group A was treated with SIT therapy and exercise intervention on the basis of routine treatment (similar to group B). The members of the two groups were evaluated before and after the experiment, and the scales were filled in under the guidance of professionals.

SIT method: According to the clinical characteristics of autism, this method is mainly embodied in exercise games. It uses games such as slides, tossing, leaping, balance beam, and sling to train children's balance, communication, and brain integration functions. While exercising, objects are required to assist each other in completing various exercise programs, thereby promoting their communication. Suitable exercise programs are selected in accordance with the conditions and hobbies of children with autism to attract their attention and promote the gradual recovery of their communication skills. Given the large number of members in the group exercise programs, more communication would be required. Patients can be guided to participate in group exercise programs to increase their mutual familiarity and to achieve therapeutic goals at the same time. These exercise programs do not only require patients to participate actively but also try to get the support of their parents. In doing so, parents can observe the gradual changes exhibited by patients. Thus, parents can adjust accordingly, and help in promoting the recovery of the patients. 


\section{Measuring Tools}

- Chinese version of CARS (Cai \& Kong 2000). The CARS scale measures 14 dimensions, including interpersonal relationships, emotional responses, adaptation to environmental changes, visual responses, anxiety responses, non-verbal communication, and so on. These dimensions measure the severity of autism according to the level of scores. Those with a total score above 30 points can be considered to have autism, whereas those with $30-36$ points are divided into mild to moderate autism. Finally, those with a score above 36 points with more than 5 indicators achieving 3 or more points can be considered to have severe autism.

- Autistic Behavior Check List (ABC List) (Lu et al. 2004). The ABC List includes 57 items, and the final score is used for evaluation. If the total score is below 31 points, then the participant is considered not to have autism at all. Those with a total score of 53-66 points can be considered to have suspected autism. If the total score is 67 points or more, then the participants can be considered to have autism.

- Curative effect index. Curative effect is assessed according to the CARS index reduction rate. If the total CARS score is more than 10 points, then the treatment is markedly effective. The treatment is considered effective at 5-10 points (including 10 points), whereas it is considered ineffective if the score is 5 points or less.

\section{Statistical Analysis}

All the data were recorded in the Epidata database, and SPSS18.0 was utilized for data analysis. The measurement data were expressed as $\mathrm{x} \pm \mathrm{S}$; Group t-test and paired t-test were employed. The difference was statistically significant at $\mathrm{P}<0.05$.

\section{RESULTS}

\section{Statistics of excluded cases}

A total of 108 cases were included in the study. During the treatment, 1 member in group B dropped out due to transfer, and 4 members in group A dropped out due to treatment difficulty caused by distant residence. These 5 cases were not statistically analyzed. A final number of 103 cases were used in the study, with 50 patients in group A and 53 patients in group B.

\section{Comparison of basic data between the two groups of children before treatment}

A total of 45 male patients and 8 female patients with an average age of $6.18 \pm 2.94$ were included in group B. A total of 43 male patients and 7 female patients with an average age of $6.17 \pm 2.44$ were included in group A (shown in Table 1). Table 2 shows that no significant difference was observed in the CARS and ABC scores between the two groups before the treatment $(\mathrm{P}>0.05)$.
Table 1. Comparison of basic data between the two groups

\begin{tabular}{lcccc}
\hline Group & $\mathrm{N}$ & Male $(\%)$ & Female $(\%)$ & Age $(\mathrm{x} \pm \mathrm{S})$ \\
\hline Group A & 50 & 43 & 7 & $6.17 \pm 2.44$ \\
Group B & 53 & 45 & 8 & $6.18 \pm 2.94$ \\
\hline
\end{tabular}

Note: $\chi 2$ test was used for comparison in gender, and $\mathrm{P}>0.05$; $\mathrm{t}$ test was conducted for comparison in age, and $\mathrm{P}>0.05$

Table 2. Comparison of CARS and ABC scores between the two groups before treatment $(\mathrm{x} \pm \mathrm{S})$

\begin{tabular}{lcc}
\hline Group & CARS & ABC \\
\hline Group A $(n=50)$ & $43.64 \pm 6.16$ & $85.72 \pm 8.68$ \\
Group B $(n=53)$ & $42.36 \pm 5.1$ & $84.74 \pm 9.32$ \\
P & 0.329 & 0.640 \\
\hline
\end{tabular}

Table 3. Comparison of CARS and ABC scores between the two groups after treatment $(\mathrm{x} \pm \mathrm{S})$

\begin{tabular}{lcc}
\hline Group & CARS & ABC \\
\hline Group A $(n=50)$ & $33.14 \pm 5.76$ & $69.92 \pm 11.52$ \\
Group B $(n=53)$ & $35.59 \pm 5.51$ & $77.56 \pm 13.31$ \\
t & -1.883 & -2.651 \\
P & 0.064 & 0.010 \\
\hline
\end{tabular}

Table 2 shows no significant difference in the CARS and $\mathrm{ABC}$ scores before the treatment $(\mathrm{P}>0.05)$.

Table 3 indicates no significant difference in the CARS and ABC scores before the treatment $(\mathrm{P}>0.05)$. After the treatment, the CARS scores reach a significant margin, and the $\mathrm{ABC}$ scores are quite significant.

Table 4 shows a comparison of the total effective rates of the two groups, indicating that $\mathrm{P}<0.05$, and the difference is statistically significant. For group A, the marked effective rate is $55.56 \%$, the effective rate is $30.56 \%$, and the total effective rate is $86.11 \%$. For group $\mathrm{B}$, the marked effective rate is $17.95 \%$, the effective rate is $46.15 \%$, and the total effective rate is $64.10 \%$ (see Table 4 for further details).

The results of the t-test in Table 5 indicate statistically significant differences in the CARS scores of the two groups before and after the treatment $(\mathrm{P}<0.05)$. Moreover, statistically significant differences were observed in the $\mathrm{ABC}$ scores of the two groups before and after the treatment $(\mathrm{P}<0.05)$.

The results of the t-test in Table 6 indicate that $\mathrm{t}=3.831$, and bilateral $\mathrm{P}=0.000<0.01$. Hence, the difference in CARS between group A and group B is considered statistically significant (i.e., group A is better than group B.) Meanwhile, $\mathrm{t}=3.492$, and bilateral $\mathrm{P}=0.001<0.01$. These results mean that the difference in $\mathrm{ABC}$ between group $\mathrm{A}$ and group $\mathrm{B}$ is considered statistically significant (i.e., group A is better than group B).

Table 7 demonstrates the autistic behavior scores of the two groups of children after three months of intervention. The sensory, language, communication, selfcare, and physical exercise ability of the experiment group significantly improved after the intervention period. Moreover, the difference with the control group after the intervention is statistically significant $(\mathrm{P}<0.01)$. 
Table 4. Comparison of curative effect index between the two groups after treatment (cases)

\begin{tabular}{lcccc}
\hline Group & $\mathrm{N}$ & Significant effect & Effective & Invalid \\
\hline Group A & 50 & 20 & 11 & 5 \\
Group B & 53 & 7 & 18 & 14 \\
Wilcoxon rank sum test & & $\mathrm{z}=-3.328$ & & $\mathrm{P}=0.001$ \\
\hline
\end{tabular}

Table 5. Comparison of CARS and ABC scores before and after treatment $(x \pm S)$

\begin{tabular}{|c|c|c|c|c|c|c|}
\hline Index & Group & Before treatment & After treatment & $\begin{array}{c}\text { Difference between } \\
\text { After and Before }\end{array}$ & $\mathrm{t}$ & $\mathrm{P}$ \\
\hline \multirow{2}{*}{ CARS } & Group A $(n=50)$ & $43.64 \pm 6.16$ & $33.14 \pm 5.76$ & $10.5 \pm 4.16$ & 15.13 & 0.000 \\
\hline & Group $B(n=53)$ & $42.36 \pm 5.1$ & $35.59 \pm 5.51$ & $6.77 \pm 4.26$ & 9.93 & 0.000 \\
\hline \multirow{2}{*}{$\mathrm{ABC}$} & Group A $(n=50)$ & $85.72 \pm 8.68$ & $69.92 \pm 11.52$ & $15.81 \pm 11.19$ & 8.48 & 0.000 \\
\hline & Group B $(n=53)$ & $84.74 \pm 9.32$ & $77.56 \pm 13.31$ & $7.18 \pm 10.21$ & 4.39 & 0.000 \\
\hline
\end{tabular}

Table 6. Comparison of differences in CARS and ABC between the two groups before and after treatment $(x \pm S)$

\begin{tabular}{llcc}
\hline Index & Group & Difference between After and Before & $\mathrm{t}$ \\
\hline \multirow{2}{*}{ CARS } & Group A $(\mathrm{n}=50)$ & $10.5 \pm 4.16$ & 3.831 \\
& Group B $(\mathrm{n}=53)$ & $6.77 \pm 4.26$ & 0.000 \\
ABC & Group A $(\mathrm{n}=50)$ & $15.81 \pm 11.19$ & 3.492 \\
& Group B $(\mathrm{n}=53)$ & $7.18 \pm 10.21$ & 0.001
\end{tabular}

Table 7. Comparison of autistic behavior scores between the two groups after intervention

\begin{tabular}{lccccc}
\hline Group & Sense & Language & Association & Life management & Somatic movement \\
\hline Group A ( $=50)$ & $16.2 \pm 0.8$ & $17.3 \pm 0.6$ & $16.5 \pm 1.0$ & $16.0 \pm 0.3$ & $15.3 \pm 1.0$ \\
Group B (n=53) & $18.2 \pm 1.0$ & $19.2 \pm 0.9$ & $18.8 \pm 1.2$ & $18.1 \pm 0.5$ & $18.3 \pm 1.1$ \\
t & -8.55 & -9.62 & -8.06 & -19.73 & -11.05 \\
P & $<0.01$ & $<0.01$ & $<0.01$ & $<0.01$ & $<0.01$ \\
\hline
\end{tabular}

\section{DISCUSSION}

\section{Analysis of the effect of SIT on the improvement of autistic symptoms in children with autism}

CARS is a professional scale for assessing the degree of autism in children. Unlike ABC, CARS needs to be assessed by trained professionals through systematic observation. No significant difference was noted in the CARS score between the two groups of children with autism before the intervention, and they all suffered a serious degree of autism. However, after 10 weeks of intervention, the CARS score of the experiment group is significantly lower than the score before the intervention. Although the results show a significant decline in the CARS score of the control group, further difference analysis suggests that the decline in the CARS score of the control group is significantly lower than that of the experiment group. As the intervention progressed, the symptoms of autism in group B also decreased. This finding indicates that routine treatment plays an important role in the recovery of children with autism. Additionally, this finding is consistent with the results of previous studies that concluded that timely and effective treatment has a positive effect on reducing autistic behaviors in children with autism (Zwaigenbaum et al. 2015, Case-Smith et al. 2015). This positive effect may be exerted through two pathways. First, drug treatment has a certain supplementary effect on autistic behaviors of children with autism (Kuhn et al. 2017).
The development of autism is particularly neurological; hence, proper use of certain neurological drugs can better prevent stereotyped behaviors of children with autism. However, the meta-analysis by (Kuhn et al. 2017) on the literature of drug treatment for autism suggests that over-reliance on drug treatment may increase side effects, which may affect overall recovery. Second, education and psychotherapy have a positive effect on improving and promoting the psychological functions of children with autism. Machalicek et al. (2016) found that interventions based on parenting behaviors can effectively improve the behavior effectiveness of children with autism. Hence, parents are important sources of social support for children with autism. Such support can further provide positive models for children with autism, enhance their internal self-efficacy, and promote their behavioral changes. In addition, one-on-one training is used in education and psychotherapy to equip children with autism with cognitive and linguistic skills and abilities, which is proven beneficial for their recovery (White et al. 2010). Compared with routine treatment, SIT is better in improving autistic symptoms in children with autism. Children with autism often appear to be overly aware of sounds and moving objects in the sensory system, which causes them to overreact to strong stimuli. Sensory system problems in children with autism can affect other aspects of their abilities, such as social skills, social input, academic performance, and so 
on (Weitlauf et al. 2017). In other words, sensory system problems are an important factor influencing other behavioral problems in children with autism. Improving the sensory system of children with autism is also an important measure to improve the overall healing effect of the treatment. In this study, we used SIT to train and improve the balance, touch, vestibule, and proprioception of children with autism through various activities including slides, swings, and skateboarding. In this way, the sensory system of children with autism can be improved, providing a basis for reducing the overall symptoms of autism (Watling \& Hauer 2015). These training activities also exercise the attention and awareness of children with autism. In the process of SIT, children with autism can perceive the positive experience brought by activities involved in the process (Wang et al. 2018).

\section{Analysis of the intervention effect of SIT on the quality of life in children with autism}

Unlike CARS, the ABC List should be filled out by parents or primary caregivers of children with autism. The behaviors of children with autism are usually examined in five main areas: feeling, communication, physical activity, language improvement, and self-care. According to this study, no significant difference was observed in the $\mathrm{ABC}$ score between the experiment group and the control group before the intervention. Nevertheless, after the three-month intervention, the ABC score of the experiment group has exhibited significantly lower score than before the intervention. However, a significant decline was observed in the ABC score of the control group. Further differential analysis suggests that the decline in the $\mathrm{ABC}$ score of the control group is significantly lower than that of the experiment group. This finding is consistent with the result of $\mathrm{Lu}$ et al. (2017). The following analysis can be given in consideration of the CARS score. Whether assessment is conducted by trained professionals or by primary caregivers of children with autism, intervention is effective to some extent. However, the decline based on the assessment performed by professionals is significantly higher than that by primary caregivers. These results suggest the importance of assessing differences. Primary caregivers of children with autism, such as parents, take a long time to get along with participants. Owing to various reasons, they suffer from low selfefficacy of parenting and high perceived psychological stress (Zaidman-Zait et al. 2017). These negative experiences may affect the objective assessment of primary caregivers on the behaviors of children with autism. Therefore, questionnaires for obtaining a variety of different data sources can be used in the future to improve the reliability of research. SIT has an important role in promoting the quality of life of children with autism. Although SIT was utilized in this study only to improve the basic sensory system functions of children with autism, this basic cognitive ability change plays an effective role in the improvement of other aspects of children with autism ( $\mathrm{Lu}$ et al. 2017). This finding is consistent with the finding of Warner et al. (2013). Additionally, the intervention model based on sensory training do not only effectively improve individuals' physical exercise ability, enhance the language cognition of children with autism and improve their self-care ability, but also promote their interpersonal status . Previous studies have shown that line-of-sight processing plays an important role in social cognition. As for children with autism, both in the laboratory scene and in the real social life scene, line-of-sight processing obstacles were common, such as not being able to establish eye contact with others and expecting others to look at the ideal goal (Tanaka \& Sung 2016). Therefore, SIT in this study can help children with autism improve their line-of-sight processing ability. Such development will enhance their social cognitive processing ability, which will then promote their social interaction ability.

\section{Comparative analysis of the intervention effects of SIT and routine treatment}

Although routine treatment can also reduce the autistic symptoms of children with autism to some extent, the curative effect that the experiment group that received SIT additionally indicated a marked effective rate of nearly $56 \%$, an effective rate of nearly $31 \%$, and a total effective rate of nearly $87 \%$. In contrast, the marked effective rate of the control group is less than $18 \%$, the effective rate is higher than that of the experiment group, and the overall effective rate is only $64 \%$. These results illustrate three issues. First, routine treatment is fundamental for the recovery of children with autism, because drug treatment, education, and psychotherapy play important roles in recovery (Xu et al. 2017). This point was also mentioned in the analysis above. Second, SIT not only directly improves the physical exercise ability, sensory ability, self-care ability of children with autism, but also enhances their social cognitive functions by improving these basic abilities, thus improving their social and language abilities (Pfeiffer et al. 2018). These results show that the behavioral characteristics of children with autism are not isolated. On the contrary, various symptoms of children with autism are interrelated; among these symptoms, the defects and deficiencies in the sensory system may be a key factor causing other problems of children with autism. Third, the reason why SIT is more effective than routine treatment probably lies in its effects in improving the basic sensory and cognitive ability of children with autism. Through the use of technologies such as balance, SIT enables children with autism to improve their sensory system functions, such as line-of-sight processing ability in continuous training, and influences changes in their psychological and social functions through changes in body functions (Robertson \& Baron-Cohen 2017). Although traditional education, psychology, and medical treatment are indispensable 
for the recovery of children with autism, achieving fundamental improvement through these trainings alone is difficult.

SIT intervention, which is athletic and game-oriented, can fully utilize the autonomy of children with autism. Children are receptive to SIT intervention, which is suitable for the physical and mental development of children with autism. In this study, a threemonth intervention in children with autism shows that SIT has a good intervention effect on the language communication and social interaction of children with autism. First, in somatosensory games, children not only have fun but also come into contact with people and the environment around them. As a result, their communication and cooperation with others are enhanced, and withdrawal behaviors are reduced. Second, children's stereotypes and problematic behaviors can be improved. For example, meaningless selfspeaking is substantially reduced after intervention, and autistic behaviors are also controlled. The stimuli from sports and games received by children with autism have a similar internal mechanism as the stimuli produced by stereotypes. They both provide children with autism a sensory stimulus of comfort, allowing them to achieve an appropriate level of excitement through sensory stimulation (Tanaka \& Sung 2016). Additionally, children can produce an excitatory sympathetic effect by participating in competitive group games (Itier \& Batty 2009). Somatosensory games, such as jumping and walking bridge games, designed in this study can also bring participants similar excitatory stimuli, indirectly improving the level of communication, self-care, feeling, language, and physical exercise abilities of children with autism.

\section{CONCLUSION}

The experiment group and the control group were compared before and after the treatment in this study to examine the effect of routine treatment and SIT on the autistic behaviors and quality of life of children with autism. The intervention effect was better on the experiment group that additionally received SIT. This study is important for the future development of SIT courses or interventions for children with autism. However, this study has limitations. Only a small number of objects were included in this study, and due to various reasons, some of the objects were lost in the later participation. These events may reduce the reliability of the study and the stability of the results. Future research, if allowed, should expand the sample size and take measures to prevent participants from withdrawing. In this study, the experiment group received not only SIT but also routine treatment. Although comparing the effects of different interventions is possible, this practice may cause interactive influence between different variables, thereby confusing the particular effect of SIT. Future studies can examine the sole effect of SIT on children with autism.

\section{Acknowledgements:}

This work was supported by National Social Sciences Fund of China (19BTY017).

Conflict of interest: None to declare.

\section{Contribution of individual authors:}

Wenxin Xu \& Jiwei Yao designed the study. Wenxin Xu wrote the manuscript.

All authors participated in the study and have approved the final manuscript.

\section{References}

1. Barnett JEH \& Crippen R: Eight steps to school-based employment training for adolescents with autism spectrum disorder and intellectual disability. Phys Disabil Educ \& Relat Serv 2014; 33:1-15

2. Bruggink A, Huisman S, Vuijk R, Kraaij V \& Garnefski N: Cognitive emotion regulation, anxiety and depression in adults with autism spectrum disorder. Res in Autism Spectrum Disord 2016; 22:34-44

3. Cai PY \& Kong KQ: A Study of Autistic Children's Behavioral Assessment and Social Cognitive Development. J Psychol Sci 2000; 23:269-274

4. Case-Smith J, Weaver LL \& Fristad MA: A systematic review of sensory processing interventions for children with autism spectrum disorders. Autism 2015; 19:133148

5. Chandler S, Howlin P, Simonoff E, O'Sullivan T, Tseng E $\&$ Kennedy J, et al.: Emotional and behavioural problems in young children with autism spectrum disorder. Dev Med Child Neurol 2016; 58:202-208

6. Cheldavi H, Shakerian S, Boshehri SNS, Zarghami M: The effects of balance training intervention on postural control of children with autism spectrum disorder: Role of sensory information. Res Autism Spectrum Disord 2014; 8:8-14

7. Chen LJ, Hu WH \& Chen M: Improving effect of sensory integration training on sensory disorder and behavioral symptoms in children with autism. Chin J Rural Med Pharm 2017; 24:37-38

8. Christensen DL, Bilder DA, Zahorodny W, Pettygrove S, Durkin MS, Fitzgerald RT, et al.: Prevalence and characteristics of autism spectrum disorder among 4-yearold children in the autism and developmental disabilities monitoring network. J Dev Behav Pediatr 2016; 37:1-8

9. Dai $X \&$ Ma TH: Effects of sensory integration training on balance and motor ability of autistic children. Chin J Rehabil Med 2008; 23:436-437

10. Dawson G, Carver L, Meltzoff AN, Panagiotides $H$, Mcpartland $J \&$ Webb SJ: Neural correlates of face and object recognition in young children with autism spectrum disorder, developmental delay, and typical development. Child Dev 2010; 73:700-717

11. White SW, Oswald D, Ollendick T, Scahill, L: Anxiety in children and adolescents with autism spectrum disorders. Clin Psychol Rev 2009; 29:216-229

12. Gates JA, Kang E \& Lerner MD: Efficacy of group social skills interventions for youth with autism spectrum disorder: A systematic review and meta-analysis. Clin Psychol Rev 2017; 52:164-181 
13. Itier RJ \& Batty M: Neural bases of eye and gaze processing: the core of social cognition. Neurosci Biobehav R 2009; 33:843-863

14. Kim $S$ \& Jeon K: Effects of a Complex Intervention Exercise Program on Lumbar Extension Strength and Stability in Female Patients with Lower Back Pain. Iran J Public Health 2017; 46:854-855

15. Kuhn M, Grave S, Bransfield R \& Harris S: Long term antibiotic therapy may be an effective treatment for children co-morbid with lyme disease and autism spectrum disorder. Med Hypotheses 2012; 78:606-615

16. Lang $R$, O'Reilly $M$, Healy $O$, Rispoli M, Lydon $H$, Streusand $W$, et al.: Sensory integration therapy for autism spectrum disorders: a systematic review. Res Autism Spect Dis 2012; 6:1004-1018

17. Li XR, Su LY, Luo XR: Field Trial Of the Revised Criteria of Mental Disorders usually first Diagnosed in Infancy, Childhood or Adolescence in CCMD-3. Chinese Mental Health $J$ 2002; 16:230-233

18. Lu JP, Yang ZW, Shu MY, Su LY: Reliability, validity analysis of the childhood autism rating scale. China $J$ Mod Med 2004; 14:119-121

19. Luxford S, Hadwin JA \& Kovshoff H: Evaluating the effectiveness of a school-based cognitive behavioural therapy intervention for anxiety in adolescents diagnosed with autism spectrum disorder. J Autism Develop Disord 2017; 47:3896-3908

20. Lu X, Wang D \& Yu D: Effect of Solution-Focused Brief Therapy-based Exercise Prescription Intervention on Adolescent Mental Health. Rev Agrent Clin Psic 2017; 26:347-355

21. Machalicek W, Lequia J, Pinkelman S, Knowles C, Raulston T, Davis T, et al.: Behavioral telehealth consultation with families of children with autism spectrum disorder: behavioral telehealth consultation. Behav Intervent 2016; 31:223-250

22. Mccoy SM, Jakicic JM \& Gibbs BB: Comparison of obesity, physical activity, and sedentary behaviors between adolescents with autism spectrum disorders and without. J Autism Develop Disord 2016; 46:2317-2326

23. Morales-Hidalgo P, Roigé-Castellví J, HernándezMartínez C, Voltas $N$ \& Canals J: Prevalence and characteristics of autism spectrum disorder among spanish school-age children. J Autism Develop Disord 2018; 48:3176-3190
24. Pfeiffer B, May-Benson TA \& Bodison SC: State of the science of sensory integration research with children and youth. Am J Occup Ther 2018; 72:7201170010p1$7201170010 p 4$

25. Robertson CE \& Baron-Cohen S: Sensory perception in autism. Nat Rev Neurosci 2017; 18:671-684

26. Tanaka JW \& Sung A: The "eye avoidance" hypothesis of autism face processing. J Autism Develop Disord 2013; 46:1-15

27. Wang Z, Mao WQ, Jin YF, Ying ZG: A Case Study of Aggressive Behavior of Autistic Children Supported by Positive Behavior. Chin Manipulation Rehabil Med 2018; 9:20-21

28. Warner E, Koomar J, Lary B, Cook A: Can the body change the score? Application of sensory modulation principles in the treatment of traumatized adolescents in residential settings. J Fam Violence 2013; 28:729-738

29. Watling $R \&$ \& Hauer S: Effectiveness of Ayres Sensory Integration and sensory-based interventions for people with autism spectrum disorder: A systematic review. Am J Occup Ther 2015; 69:6905180030p1-6905180030p12

30. Weitlauf AS, Sathe N, Mcpheeters ML \& Warren ZE: Interventions targeting sensory challenges in autism spectrum disorder: a systematic review. Pediatrics 2017; 139:e20170347

31. Wu JZ: A survey of sensory integrative dysfunction and attention deficit and hyperactivity in 56 children with autism. Chinese J Pract Pediatr 2011; 26:203-206

32. Xu WX, Li ML, Yao JW: Intervention of Collective Exercise on the Mental Health of Elderly Hypertensive Patients. Iran J Public Health 2016; 45:314-321

33. Xu WX, Zhao ZJ, Ceng MJ, Yao JW: Intervention Effect of the Exercise-based Rehabilitation Model on the Loneliness and Health Behavior of Adolescents with Autism. Rev Agrent Clin Psic 2018; 27:455-465

34. Zaidman-Zait A, Mirenda P, Duku E, Vaillancourt T, Smith IM, Szatmari $P, \ldots \&$ Zwaigenbaum L: Impact of personal and social resources on parenting stress in mothers of children with autism spectrum disorder. Autism 2017; 21:155-166

35. Zwaigenbaum L, Bauman ML, Choueiri R, Kasari C, Carter A, Granpeesheh D, ... \& Pierce K: Early intervention for children with autism spectrum disorder under 3 years of age: recommendations for practice and research. Pediatrics 2015; 136(Suppl 1):S60-S81

Correspondence:

Jiwei Yao

Physical Education Institute, Hunan University of Science and Technology,

Xiangtan, Hunan, 411201, China

E-mail: yjwdxs09141020@163.com 